\title{
Towards a Visible Light Network Architecture for Continuous Communication and Localization
}

\author{
Jialiang Zhang, Chi Zhang, Xinyu Zhang and Suman Banerjee \\ University of Wisconsin - Madison \\ jialiang.zhang@ece.wisc.edu, czhang296@wisc.edu, xyzhang@ece.wisc.edu, suman@cs.wisc.edu
}

\begin{abstract}
Visible light communication (VLC) technology piggybacks on legacy illumination infrastructure, and creates a communication channel by modulating LEDs' light emission patterns. Existing VLC research mostly focused on improving single link communication capacity, or localizing a single static handset equipped with light sensors. In this paper, we advance this technology to the next layer, and propose an integrated visible light network (VLN) architecture that aims to realize continuous communication coverage and location tracking for indoor mobile devices. Our VLN architecture uses a powerline backbone together with a backend server to centrally control and coordinate ceiling-mounted LED access points (APs). The LED APs are dynamically clustered depending on the client location, and tightly synchronize and cooperate with each other, in order to enhance link quality and spatial diversity, thus smoothing the client's transition between cells and alleviating the impact of device motion, body shadowing, etc. . We have prototyped the VLN architecture using customized hardware platform and an entire software-defined network stack. Preliminary results demonstrate the feasibility of the VLN concept, and potential of the architectural design.
\end{abstract}

\section{CCS Concepts}

-Computer systems organization $\rightarrow$ Special purpose systems; $\bullet$ Hardware $\rightarrow$ Signal processing systems; •Information systems $\rightarrow$ Location based services; $\bullet$ Human-centered computing $\rightarrow U$ Ubiquitous and mobile computing systems and tools;

\section{Keywords}

Visible light communication; Visible light networks; Visible light localization; Signal processing

\section{INTRODUCTION}

The proliferation of LEDs are driving smart lighting technologies that piggyback visible light communication (VLC) capability on illumination infrastructure. VLC uses LEDs as transmitter and light sensors (e.g., photodiode or camera) as receiver, establishing com-

Permission to make digital or hard copies of all or part of this work for personal or classroom use is granted without fee provided that copies are not made or distributed for profit or commercial advantage and that copies bear this notice and the full citation on the first page. Copyrights for components of this work owned by others than ACM must be honored. Abstracting with credit is permitted. To copy otherwise, or republish, to post on servers or to redistribute to lists, requires prior specific permission and/or a fee. Request permissions from permissions@ acm.org.

VLCS'16, October 03-07, 2016, New York City, NY, USA

(c) 2016 ACM. ISBN 978-1-4503-4253-7/16/10 . \$ $\$ 15.00$

DOI: http://dx.doi.org/10.1145/2981548.2981556 munication link by "flickering" at a speed unperceivable to human eyes. The IEEE 802.15.7 standard has established a cornerstone for VLC MAC/PHY protocols, allowing point-to-point bit-rate of up to $96 \mathrm{Mbps}$. In addition, VLC affords a more usable and reliable indoor localization [1,2] than RF technologies, because VL signals propagate in a more deterministic way than radio signals. Combining the communication and localization capabilities, VLC can enable a new line of location-dependent "infocast" applications in indoor environment.

Prior research in VLC has focused a lot on single point-to-point communication using visible light as medium [3]. However, in this paper, we argue that in addition to providing yet another communication substrate, visible light provides new opportunities for data connectivity and mobile applications that are not readily available in other communication technologies. For example $(i)$ it provides the ability to send high volume and high bitrate downlink data from infrastructure to mobile clients in indoor environments while keeping energy consumption low and (ii) it holds potential for continuous location tracking for mobile devices.

To realize such capabilities, a point-to-point VLC is not adequate and one needs to explore various design considerations at the next layer up. This motivates a vision of Visible Light Networking (VLN) which provides necessary coordination across VL access points (APs) and clients for seamless data communication and localization under mobility settings. To this end, we explore a VLN architecture that connects VL APs with a powerline communication (PLC) backbone, as it is natural and available in indoor environment supporting lighting infrastructure. Figure 1 illustrates this VLN architecture. The front-haul comprises ceiling-mounted LED fixtures, acting as APs for roaming clients indoor. Via the PLC backhaul, a central server connects with all APs, schedules their transmissions, and acts as gateway that stores and forwards downlink data to the APs. The clients receive downlink data from the APs via VL links, while using a low-rate WiFi interface as uplink data/signaling channel.

Such an architecture offers several immediate advantages over a barebone VLC or WiFi network: (i) it overcomes the inability of VLC APs to directly communicate/coordinate with each other (as they tend to face downwards and not towards each other); (ii) it is backward compatible with existing infrastructure, evading the additional cabling cost of a separate backhaul. (iii) it supports locationdependent mobile applications that require high downlink capacity, high energy-efficiency, and low uplink capacity. Clients running such applications can be moving machines (e.g., robots) or mobile devices (e.g., smartphones, smart glasses, VR headset) with a light sensor and held by human users.

Atop the VLN architecture, we design a connectivity management framework to support continuous link connection and realtime location tracking. Conceptually, we deem the ceiling-mounted 


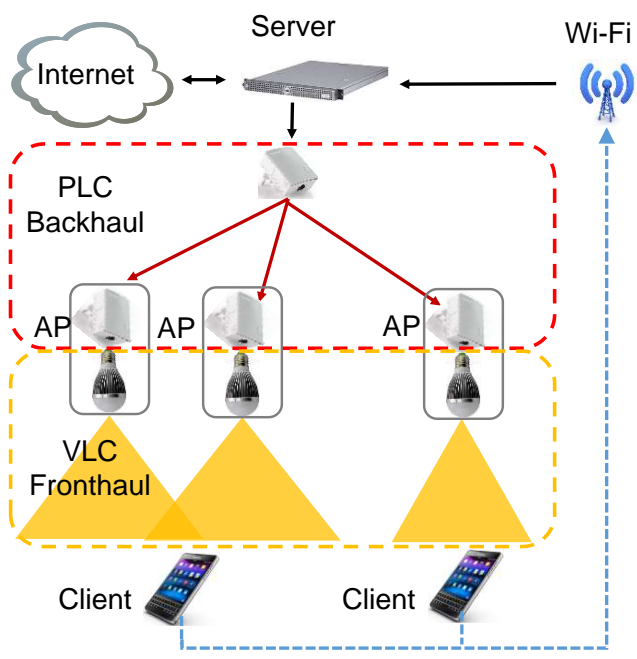

Figure 1: VLN system architecture.

APs as distributed antennas wired through the powerline and centrally managed by the server. The server follows several principles to achieve interruption-free communication/localization. (i) $D y$ namic clustering. Depending on a client's location, the server dynamically assigns it a primary AP, together with multiple "scouting APs". These APs together form an AP cluster and serve the client through cooperative diversity and spatial diversity, thus smoothing out link dynamics. (ii) Soft binding. A client associates to the server instead of individual APs, and thus no reassociation or connection reestalibshment is needed as it roams across APs or clusters. (iii) Low-rate but high reliability uplink. Our target applications are downlink-dominated, requiring intermittent but highly reliable uplink feedback. The WiFi interface, used at low-rate and proving much wider coverage, naturally serves for this purpose. (iv) Leveraging AP cooperation for real-time localization. The ceiling mounted APs have a fixed spatial relation known to the server. Also, the AP-to-client VL channel has a fairly deterministic coverage and propagation profile. Thus, by allowing clients to collect beacons from AP clusters and individual APs, the server can easily realize continuous tracking with sub-cell level granularity. Such localization support can be much more reliable and usable than RF based approaches.

We validate the VLN architecture by prototyping an entire network stack from PHY layer communication hardware to application layer protocols. The prototype realizes the VL transceiver hardware, PLC-to-VLC interface, downlink-uplink integration, along with basic server-side scheduling functionalities. Our preliminary evaluation demonstrates the feasibility of an integrated VLN architecture, along with benefits from AP coordination and centralized scheduling.

The concept of VLC and PLC integration was raised a decade ago [4]. However, to our knowledge, our work represents the first to leverage this integrated architecture for large-scale VLN that supports location-dependent mobile applications. Our specific contributions include:

- Proposing a complete VLN architecture which enables tight coordination between VL APs to harness cooperative diversity and spatial diversity.

- Incorporating a connectivity management framework into the VLN architecture, with mechanisms such as dynamic clustering and soft binding to facilitate continuous communication and location tracking.

- Implementing VLN architecture with customized VLC hard- ware drivers and software-defined network stack. Conducting preliminary evaluation of the VLN architecture and identifying potentials, tradeoffs and limitations.

\section{BACKGROUND AND MOTIVATION}

In this section, we first review background of point-to-point visible light communication technologies. Then we introduce the application scenarios of a large-scale VLN architecture, along with the challenges in realizing continuous communication/localization within the VLN.

\subsection{A Primer on Point-to-Point Visible Light Communications (VLC)}

VLC offers compelling communication and location sensing features to supplement conventional RF technologies like WiFi. A key advantage of VLC is that it enables communication/sensing by retrofitting existing LED fixtures, without affecting their primary illumination function: VLC uses intensity modulation which only varies signal magnitude, at a rate beyond human perception. A VLC receiver can either use a light-sensor or camera as frontend, both widely available in modern mobile devices. Besides, the following properties make VLC particularly attractive for handheld devices in indoor environment.

Deterministic propagation enables reliable localization. VLC highly relies on the directional LOS channel, because visible light signals have nano-meter wavelength, and hence the reflection, diffraction and diffusion effects are negligible. As a result, the LOS signal attenuation follows a deterministic function with distance. Taking advantage of this property, existing works $[1,2]$ realized point-wise localization of VLC devices, with sub-meter level precision.

Low transceiver complexity and zero spectrum cost. Current VLC transceivers use magnitude modulation, thus evading sophisticated baseband processing units, and front-end circuits like frequency mixers or phase estimation. In addition, the visible light spectrum is unlicensed and largely unused for communication. Availability of this free spectrum is another compelling reason for practical usage.

High power efficiency. A ceiling-mounted LED transmitter reuses the illumination power for VLC, and only requires a very lowpower microcontroller circuit to modulate the "flickering". The receiver front-end, owing to simplified circuitry, only consumes 7.5 $\mathrm{mW}$ [5] even when sampling at $20 \mathrm{Msps}$ rate. A Wi-Fi front-end would consume 150 to $300 \mathrm{~mW}$ [6] at a similar sampling rate.

\subsection{From VLC to VLN}

We propose to leverage the aforementioned salient properties to realize large-scale VLN, which could be deployed in indoor environment with pervasive LEDs on the ceiling. Example scenarios include shopping malls, grocery store, cafeteria, conference room, etc., as shown in Figure 2. Modern commercial buildings typically require continuous lighting over space, and thus amenable to be retrofitted with VLN.

\subsubsection{VLN Applications}

VLN targets location-dependent indoor mobile applications that require both communication and reliable localization support, at low cost and high energy efficiency. Below we discuss several niche applications of VLN.

Location-based advertisement. For the retail sector, up to $70 \%$ of sales are made at the aisle level [7], where the location-based advertisement can be a highly lucrative value proposition, with anticipated market value of $\$ 5$ billion by 2018. Despite the vast literature of $\mathrm{WiFi}$ localization, the stability and usability remains as 


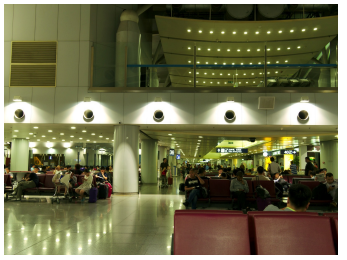

(a)

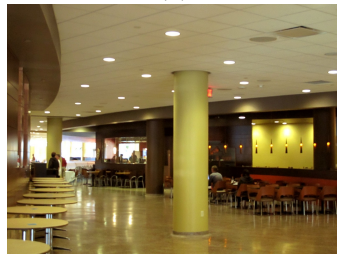

(c)

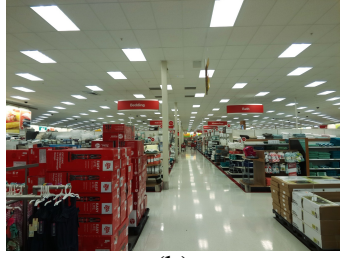

(b)

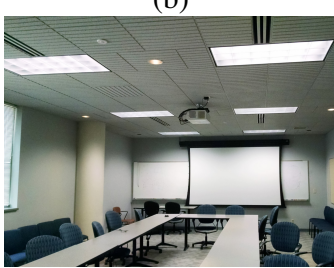

(d)
Figure 2: Potential deployment scenarios of VLN: (a) Airport. (b) Grocery store. (c) Cafeteria. (d) Conference room.

open challenges. Owing to highly regular coverage and propagation of LEDs, VLN easily overcomes such problems. Ceiling-mounted VLN APs can stream video/audio/images to clients at specific locations, to recommend products and provide informative purchase guidance.

Infostations in public spaces. In museums, convocation centers, or other public exhibitions, VLN APs can serve as real-time infostations, automatically localizing users and delivering audiovisual information to users' mobile devices or specialized handsets/headsets.

Virtual-reality and wearable applications. A virtual reality headset often needs to localize itself in real-time, while downloading videos/images from a backend server. VLN can provide such localization and data delivery services inside game stores, exhibition rooms, etc. Similarly, VLN can serve wearable devices like smart watches, smart glasses, and smart jewelry, as long as such devices are equipped with a light sensor and covered by LED illumination when localization/communication services are needed.

Robotic navigation indoor. VLN is particularly useful for navigating robots, which may be mobile but can maintain consistent light exposure. Such robotic applications can exist in factory warehouse, as well as modern exhibition rooms.

\subsubsection{Challenges Towards Continuous VLN Cover- age}

To support the aforementioned applications, a VLN client needs to have a receiver interface with a light-sensor, and needs to be exposed under LED illumination. These requirements are naturally satisfied in target usage scenarios with uniform LED illumination and battery-powered client devices.

The key challenge of VLN lies in the infrastructure side, i.e., how to manage and coordinate the LED APs for interruption-free communication coverage and real-time localization. Specifically, (i) The APs cannot directly communicate with each other. Existing VLC standards like 802.15.7 [3] assumes all transmitters have light-sensors and LEDs facing each other, which is unrealistic for ceiling-mounted LEDs. (ii) The LOS propagation nature of visible light signals removes multipath diversity, rendering AP-to-client links highly vulnerable to blockage, body shadowing and clients' orientation variation. (iii) At coverage boundaries, visible light signals may become too weak to support either localization or communication functionalities. (iv) Each LED has a very limited coverage. Thus, the VLN needs to handle frequent handoff between cells for both communication and localization scenarios.

\section{VLN ARCHITECTURE DESIGN AND IM- PLEMENTATION}

We propose a complete VLN solution to meet the aforementioned challenges. Our key idea is a two-tired network architecture integrating a PLC backhaul with VLC fronthaul, as shown in Figure 1. Based on this architecture, we design and implement a centralized connectivity management framework to support continuous coverage and real-time localization services.

A typical workflow of the VLN architecture is executed as follows. When a client device roams into the VLN's coverage space, it requests association with the back-end server by using the ambient WiFi as uplink signaling channel, and nearby LED AP for downlink. Afterwards, the server continuously tracks the client location based on the visible light signal strength it senses from the nearest AP called primary AP. Meanwhile, the server forces the primary AP's one-hop neighbors (called scouting APs) to assist the primary $\mathrm{AP}$, which together form a "distributed antenna cluster", to ensure continuous coverage for communication and location sensing.

Below we detail the essential components and design choices in realizing this VLN workflow.

\subsection{Network Architecture Design}

PLC backhaul for connectivity management. The PLC backhaul connects all LED APs to the server, a central controller that maintains precise synchronization between APs for cooperative communication, and delivers downlink data to each AP. Each AP maintains a separate PLC interface and MAC identity addressable by the server.

We note that an indoor powerline network usually maintains a tree structure: wires branch off a breaker box and are distributed towards different rooms. This entire tree structure can be considered as an "Ethernet" with a single contention domain, where PLC devices connected to outlets can communicate with each other at hundreds of Mbps rate [8]. For larger buildings containing multiple breaker boxes, a higher level of gateway is needed at each breaker box.

"Distributed antenna" architecture with dynamic clustering. The primary and scouting APs together form an AP cluster with tight waveform-level synchronization to enable cooperative communication. These APs are assigned dynamically by the server based on the client's location. This "distributed antenna" architecture offers a number of salient advantages: (i) It creates a virtual AP with large coverage, thus smoothing the transition between cells as the client moves. (ii) PHY layer cooperation between the APs enhances link quality especially at cell boundaries. Meanwhile, spatial diversity of APs reduces the risk of body shadowing on the VLC client devices.

Soft binding. Instead of bound to individual APs, each client only needs to run a one-time association to the server that controls the entire VLN. Such a soft binding mechanism eliminates the frequent handoffs in conventional multi-cell wireless network architecture. In effect, together with dynamic clustering, soft binding ensures a client never has to run any handoff signaling as it roams across the APs.

Centralized scheduling. Since the ceiling mounted LED APs are usually out of each other's FOV, the standard CSMA based MAC (e.g., the IEEE 802.15.7) is no longer applicable to our VLN architecture. We thus adopt a location-aware TDMA scheduling protocol centrally run by the server. The schedule is dispatched on a super-frame basis. Each super-frame lasts for a few data frames, during which the server simultaneously delivers different data to different AP clusters covering different clients.

The uplink channel only requires low-rate intermittent acknowl- 
edgement frames and other signaling messages, but needs high reliability guarantee. VLC is no longer suitable as the uplink because a mobile LED transmitter causes uncomfortable effects on human users and more importantly, it may suffer from the same reliability problem as downlink, occasionally causing both downlink data and uplink signaling channel to fail. Therefore, we use the clients' WiFi interface to establish a wider-coverage, low-rate uplink to the server.

AP cooperation for interruption-free real-time localization. With the VLN architecture, we can easily extend existing staticclient localization schemes $[1,2]$ to support continuous tracking. The dynamic AP clustering mechanism ensures a client can receive strong beacons even under the risk of blockage/shadowing. A simple beaconing mechanism, with each AP cluster sending a single beacon and individual APs following up, can allow each client to achieve sub-cell level localization accuracy based on APs' known location. More sophisticated algorithms that leverages VLC propagation model may achieve higher accuracy, but are left for our future work.

\subsection{VLN System Implementation}

Consistent with Figure 1, our implementation consists of three main modules: server, AP and user equipment (UE).

The server, which is a PC workstation running Linux, acts as the central controller as well as the Internet gateway. We have implemented the location-based centralized scheduling, soft binding, and cell-level localization at the server side. We use a commercial PLC-to-Ethernet adapter [8] to interface the server with the powerline, and implement the forwarding functions needed to deliver data to the LED APs.

The AP, which comprises a BeagleBoneBlack board (BBB) and a customized VL transmitter, bridges the VL front-haul and PLC back-haul. Figure 3 illustrates the LED AP that we have developed. The AP receives data frames from the server, and then transmits the data immediately through the LED front-end. If the server does not receive an uplink ACK in time, it reschedules and retransmits the data frame. Since the server has precise information about transmission schedule and duration, the APs only act as dumb relays and need not even buffer data. In addition, we have integrated the PTP driver [9] (originally used for precise synchronization between Ethernet clients) on the AP and server, to ensure all APs act synchronously upon receiving data frames from the server.

The UE converts received light into voltage signals, which are sampled by a 10-bit ADC and processed by the BBB to obtain the light intensity. We have implemented a simple OOK modulation mechanism between the AP and UE. Section 4 profiles the hardware capability of our prototype. The UE also has a Wi-Fi interface, used to transmit the uplink data as well as the channel feedback.

We have also integrated the communication hardware as a PHYlayer interface into the Linux network stack. The integrated software driver allows us to support native network applications with either TCP or UDP traffic.

\section{PRELIMINARY RESULTS}

In this section, we conduct a basic profiling of the VLN prototype, and then verify the feasibility and effectiveness of the AP clustering mechanism. We also perform an integrated test through end-to-end TCP/UDP data transfer. The LED cell-based localization mechanism is straightforwardly realized and omitted due to space constraint. Our experimental environment is illustrated in Figure 4. We have built 5 APs and 1 UE. Each VLN AP is placed on top of a lighting pole at $2.8 \mathrm{~m}$, with $1.8 \mathrm{~m}$ vertical distance to the UE.

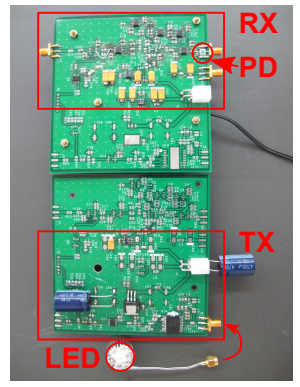

Figure 3: AP and UE devices that we have developed for testing VLN.

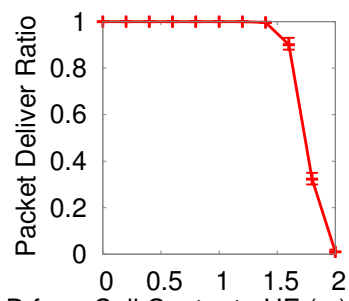

$\mathrm{D}$ from Cell Center to UE $(\mathrm{m})$

Figure 5: Packet Deliver Ratio at different distance

\subsection{Basic Profiling}

AP Coverage. We evaluate the relationship between packet delivery ratio (PDR) and the horizontal distance between AP and UE. Figure 5 shows that the PDR keeps above $95 \%$ within a radius of $1.5 \mathrm{~m}$, but drops fast beyond that distance. The coverage radius is small, mainly due to the low transmission power ( $2 \mathrm{~W}$ ) of our prototype AP. As transmission power increases, the coverage radius will increase substantially (following an inverse-square scaling law [10]).

AP synchronization. Recall APs within the same cluster need to synchronize their transmission at symbol level to harness cooperative diversity. We measure the synchronization accuracy by connecting two APs' front-end output to an oscilloscope. We capture 1000 synchronized transmissions from these APs (both connected to and controlled by the server via PLC backbone), and then measure the skew of the beginning of each packet. The CDF plot in Figure 6 shows that the 90 percentile synchronization error is less than $10 \mu s$. The synchronization is not perfect, and may cause nearby symbols to partially overlap. To tame such negative effects, we empirically constrain the overlapping to be less than $10 \%$ of symbol duration. So, the symbol duration should be longer than $100 \mu \mathrm{s}$, which corresponds to a bit-rate of $10 \mathrm{kbps}$.

Breakdown of communication/processing latency. We measure the latency of the main modules inside the VLN architecture and summarize the results in Table 1. The latency bottleneck lies in the WiFi uplink, which can become congested in a busy radio environment. The VL link latency is mainly attributed to the transmission delay: our current prototype has a maximum rate of 10 Kbps and frame size of 1024 bits, translating into a latency of 103 $\mathrm{ms}$. The MAC/PHY processing on BBB costs about $2 \mathrm{~ms}$ at both the UE and AP. With a dedicated baseband processing unit, we expect to achieve a rate comparable to the 802.15 .7 specification (up to 96 Mbps).

\subsection{AP clustering}

We first use numerical simulation to show the potential bene- 
Table 1: Latency of our VLN implementation.

\begin{tabular}{|c|c|}
\hline Component & Latency \\
\hline WiFi uplink (Idle) & $87 \mathrm{~ms}$ \\
\hline WiFi uplink (Busy) & $211 \mathrm{~ms}$ \\
\hline VL downlink & $103 \mathrm{~ms}$ \\
\hline BBB processing time & $2 \mathrm{~ms}$ \\
\hline
\end{tabular}

fits from dynamic AP clustering. The simulation uses a standard VLC channel model [10], and assumes OOK transmission from APs with perfect synchronization (and hence received power levels linearly add up). We investigate the minimum deployment density to eliminate black spots, ensuring a client can decode frames even at cell boundary. For simplicity, we focus on two-AP case. From the simulation results in Figure 7 (a), we see that the minimum separation between APs increases sublinearly as transmit power increases. More interestingly, with AP clustering and cooperation, the required density can be relaxed substantially compared with the non-cooperative case.

Figure 7 (b) shows that coordinated transmission can make the cell boundary receiver less sensitive to the orientation variation-a critical problem in practical usage scenarios. With uncoordinated APs, the maximum tolerable rotation angle of cell edge UEs keeps decreasing when we use larger transceiver gain (higher transmission power and receiver sensitivity) to expand coverage. In contrast, with coordinated APs, the maximum tolerable angle follows an increasing trend, implying that the device can survive fierce rotation, movement, or other link dynamics.

We now use our testbed to test the SNR improvement by enabling AP clustering and coordination. 4 APs are placed at corner positions $(0,0),(4,0),(0,4)$ and $(4,4)$. Figure $7(\mathrm{c})$ plots the measured RSS improvement for UEs at different locations in between, compare to a baseline where a single AP at $(0,0)$ is activated. We see the UE's RSS can be improved at almost every location, with up to $3 \mathrm{~dB}$ gain (corresponding to combined power from two nearest APs). This power gain, plus the spatial diversity from multiple APs, can substantially reduce the impact of link dynamics caused by device mobility, orientation variation, body shadowing, etc. Note that, since the cell coverage is smaller than the AP separation, there is nearly no RSS gain at $(0,0)$.

\subsection{Network-level Gain from Coordination}

We evaluate the entire VLN stack by putting all the implemented components together, and run a TCP file transfer between the server and client. We vary the AP cluster's size from 1 to 5, while keeping the client roughly within the cluster's coverage. Figure 7(e) plots the TCP throughput with and without AP cooperation (error bar represents std. across $20 \mathrm{UE}$ locations). We observe that the throughput gain from cooperation increases as we increase the number of APs, and goes up to $34 \%$ with 5 APs. The absolute throughput value is low, ranging from $3 \mathrm{Kbps}$ to $20 \mathrm{Kbps}$, because our hardware platform only supports $10 \mathrm{Kbps}$ for a single link.

\section{DISCUSSION AND FUTURE WORK}

\subsection{Limitations and Performance Scalability}

Compared with conventional RF communication network, VLN bears a number of limitations. First, the VL link only works in line-of-sight and can serve mobile devices only when they are exposed under the LED lights. Second, to achieve continuous coverage, VLN requires a reasonably dense LED deployment (a few meters of inter-LED distance), which is not applicable for home environment. Due to such limitations, VLN cannot supercede WiFi, and should only complement it in niche applications with strong localization requirements (Section 2.2.1).
Our current VLN implementation has limited coverage and data rate. The coverage is constrained by the LED transmission power and the sensitivity of photodiode sensor. The data rate is limited by the processing speed of BBB micro-controller and the softwarebased PTP synchronization accuracy. Thus, the PHY layer performance of the implementation can be scaled with a high power LED, high-speed controller and hardware PTP synchronization. For example, with a full-fledged implementation, a $10 \mathrm{~W}$ LED could provide a coverage of $5 \mathrm{~m}$ radius [11], which can significantly reduce the LEDs' deployment density requirement. Also, link throughput of more than $100 \mathrm{Mbps}$ can be achieved with higher LED transmission power [12], OFDM modulation [12,13] and proper equalization at receiver side [14]. However, our current implementation is mainly used to verify the VLN architecture and design components. Improving absolute throughput values is beyond our scope of research.

\subsection{Future Work}

Dynamic coverage adaptation. AP clustering/cooperation expands coverage, but may generate more inter-cluster interference and reduce network throughput when multiple users coexist. To balance this tradeoff, we can adaptively control the cluster size and transmission power levels on a per-frame basis. Such fine-grained power control has shown to be infeasible for practical WiFi networks due to multipath reflections, but it holds great potential for VLN which works in LOS and has a highly regular propagation range.

Continuous, real-time location tracking. Our current system only has a straightforward cell-level localization mechanism. As future work, we will design a fine-grained localization scheme that takes advantage of (i) known and regular spatial layout of APs, (ii) motion sensors on VL receivers, (iii) spatial-temporal cooperation between APs that the client consecutively passes through.

Location assisted predictive scheduling. Given the location information, the server can schedule the AP clustering in a more finegrained manner (instead of invoking all one-hop neighbors as in our current implementation), thus making a better tradeoff between cooperation gain and inter-cluster interference.

Full-fledged implementation. Our current implementation realizes a basic network stack from PHY up to application layers. We plan to implement the above extended designs and conduct a comprehensive system level test to demonstrate the potentials of VLN.

\section{RELATED WORK}

VLC technologies and applications. VLC concept originates from optical wireless technology, which has been extensively studied from communications perspective, with an emphasis of improving link bit-rate. Development along this line culminated in the IEEE 802.15.7 standard, with a bit-rate of $11.67 \mathrm{Kbps}$ to $96 \mathrm{Mbps}$ using variants of $\mathrm{OOK}$ modulation. Alternative hardware setup uses cameras as receivers $[2,15,16]$, albeit at much lower rate (below 1 Kbps) due to low sampling rate of COTS cameras. Recent research has also piggybacked on VLC systems to enable novel sensing applications, e.g., sensing finger position and enabling new modalities of mobile interaction [17].

Relatively less research has touched upon VL network protocols at MAC layer and above. The 802.15.7 ratified a CSMA primitive in 2011, yet it remains open how well such a protocol performs in practice, especially considering potential link dynamics. Alternative protocols like VICO [18] and Firefly [19] schedule transmissions in a static VL system, and improves capacity by tunning beamwidth and direction. But these protocols do not address the VL link dynamics. DLit [20] adapts VLC link bit-rate based on 

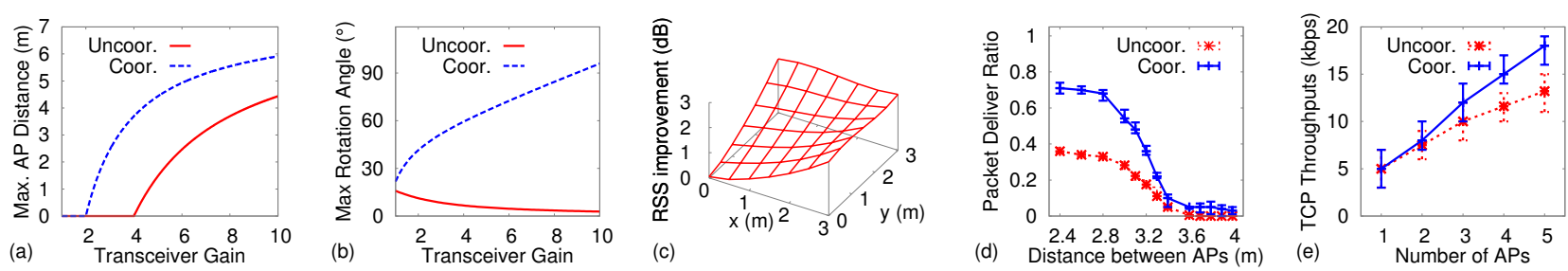

Figure 7: (a) Maximum distance between AP versus transceiver gain $\left(\frac{\mathrm{TX} \text { Power }}{\mathrm{Rx} \text { Sensitivity }}\right)$. (b) Maximum tolerable client rotation to maintain connectivity. (c) RSS improvement by 4 AP coordination. (d) PDR of coordinated and uncoordinated VLN. (e) TCP performance of coordinated and uncoordinated VLN.

sub-frame level SNR estimation, but works only in a single cell.

Integration of VLC and PLC. The idea of integrating VLC and PLC was pioneered by Komine et al. [4]. Point-to-point prototype implementation has been demonstrated that realizes analog PLCVLC interface, i.e., baseband signals on PLC directly control the On-Off modulation on the VL front-haul $[16,21]$. More recently, Kuo et al. [22] proposed a software-based control plane for such a network architecture, along with potential applications in mobile sensing. However, to our knowledge, no existing work has thoroughly addressed the problem of robust connectivity in a multi-cell VLC network, with or without a PLC backbone.

Seamless connectivity in wireless networks. Seamless connectivity is a classical issue in wireless networks. A celebrated solution is soft-handoff $[23,24]$, or coordinated multipoint transmission [25], which allows a cell-edge mobile device to be served by multiple nearby basestations. Starfish's high-level principle bears similar spirit with soft handoff, but the unique characteristics of VL channel lead to new challenges and opportunities, as discussed in Section 1 and 2.

\section{CONCLUSION}

This paper proposes a visible light network (VLN) architecture that expands the single-link visible light communication technology to network level. We develop a two-tier architecture using a powerline network as backbone to tightly synchronize and coordinate ceiling-mounted LED transmitters which serve mobile devices equipped with light sensors. We have prototyped the VLN architecture through customized hardware and a complete softwaredefined network stack. Preliminary results demonstrate the potential of VLN in realizing continuous coverage and supporting location-dependent indoor mobile applications.

\section{Acknowledgements}

We appreciate the insightful comments and feedback from the anonymous reviewers. The work reported in this paper is supported in part by the NSF under Grant CNS-1506657, CNS-1518728, CNS1343363, and CNS-1350039.

\section{REFERENCES}

[1] L. Li, P. Hu, C. Peng, G. Shen, and F. Zhao, "Epsilon: A Visible Light Based Positioning System," in Proc. of USENIX NSDI, 2014.

[2] Y.-S. Kuo, P. Pannuto, K.-J. Hsiao, and P. Dutta, "Luxapose: Indoor positioning with mobile phones and visible light," in Proc. of ACM MobiCom, 2014.

[3] H. Elgala, R. Mesleh, and H. Haas, "Indoor Optical Wireless Communication: Potential and State-of-the-Art," IEEE Communications Magazine, vol. 49, no. 9, 2011.

[4] T. Komine and M. Nakagawa, "Integrated System of White LED Visible-light Communication and Power-line Communication," IEEE Trans. on Consumer Electronics, vol. 49, no. 1, 2003.

[5] Glen Brisebois, "100MHz Op Amp Features Low Noise Rail-to-Rail Performance While Consuming Only 2.5mA," 2011. [Online]. Available: http://cds.linear.com/docs/en/design-note/dn308f.pdf
[6] C. Zhang, X. Zhang, and R. Chandra, "Energy Efficient WiFi Display," in ACM MobiSys, 2015.

[7] ABI Research, "Indoor Location in Retail: Where Is the Money?" 2010.

[8] TP-Link, “AV200 Nano Powerline Adapter Starter Kit." [Online]. Available: http: //www.tp-link.com/en/products/details/cat-18_TL-PA2010KIT.html

[9] K. Correll, N. Barendt, and M. Branicky, "Design considerations for software only implementations of the ieee 1588 precision time protocol," in Conference on IEEE, vol. 1588, 2005, pp. 11-15.

[10] Z. Ghassemlooy, W. Popoola, and S. Rajbhandari, Optical Wireless Communications: System and Channel Modelling with MATLAB. CRC Press, 2012.

[11] K. Lee, H. Park, and J. Barry, "Indoor Channel Characteristics for Visible Light Communications," IEEE Communications Letters, vol. 15, no. 2, 2011

[12] H. Elgala, R. Mesleh, and H. Haas, "Indoor Broadcasting Via White LEDs and OFDM," IEEE Trans. on Consumer Electronics, vol. 55, no. 3, 2009.

[13] O. Gonzalez, R. Perez-Jimenez, S. Rodriguez, J. Rabadan, and A. Ayala, "OFDM Over Indoor Wireless Optical Channel," IEE Proceedings on Optoelectronics, vol. 152, no. 4, 2005.

[14] H. L. Minh, D. O’Brien, G. Faulkner, L. Zeng, K. Lee, D. Jung, Y. Oh, and E. T. Won, "100-mb/s nrz visible light communications using a postequalized white led," Photonics Technology Letters, IEEE, vol. 21, no. 15, pp. 1063-1065, Aug 2009.

[15] S. D. Perli, N. Ahmed, and D. Katabi, "PixNet: Interference-free Wireless Links Using LCD-camera Pairs," in Proc. of ACM MobiCom, 2010.

[16] N. Rajagopal, P. Lazik, and A. Rowe, "Visual Light Landmarks for Mobile Devices," in Proc. of ACM/IEEE IPSN, 2014.

[17] C. Zhang, J. Tabor, J. Zhang, and X. Zhang, "Extending Mobile Interaction Through Near-Field Visible Light Sensing," in ACM MobiCom, 2015.

[18] Z. Xu, S. V. Krishnamurthy, K. Pelechrinis, J. Ning, Y. Li, and L. Wang, "VICO: A Framework for Configuring Indoor Visible Light Communication Networks," in Proc. of IEEE International Conference on Mobile Ad-Hoc and Sensor Systems (MASS), 2012.

[19] N. Hamedazimi, Z. Qazi, H. Gupta, V. Sekar, S. R. Das, J. P. Longtin, H. Shah, and A. Tanwer, "FireFly: A Reconfigurable Wireless Data Center Fabric Using Free-space Optics," in Proc. of ACM SIGCOMM, 2014

[20] J. Zhang, X. Zhang, and G. Wu, "Dancing with Light: Predictive In-frame Rate Selection for Visible Light Networks," in Proc. of IEEE INFOCOM, 2015.

[21] P. Amirshahi and M. Kavehrad, "Broadband Access Over Medium and Low Voltage Power-Lines and Use of White Light Emitting Diodes for Indoor Communications," in IEEE CCNC, vol. 2, 2006.

[22] Y.-S. Kuo, P. Pannuto, and P. Dutta, "System Architecture Directions for a Software-defined Lighting Infrastructure," in Proc. of ACM Workshop on Visible Light Communication Systems, 2014.

[23] S. Seshan, "Low-latency Handoff for Cellular Data Networks," Ph.D. dissertation, 1995.

[24] D. Wong and T. J. Lim, "Soft Handoffs in CDMA Mobile Systems," IEEE Personal Communications, vol. 4, no. 6, 1997.

[25] Patrick Marsch (Editor) and Gerhard P. Fettweis (Editor), Coordinated Multi-Point in Mobile Communications: From Theory to Practice. Cambridge University Press, 2011. 